\title{
AN INCIDENCE CONJECTURE OF BOURGAIN OVER FIELDS OF POSITIVE CHARACTERISTIC
}

\author{
JORDAN S. ELLENBERG ${ }^{1}$ and MÁRTON HABLICSEK ${ }^{2}$ \\ ${ }^{1}$ Department of Mathematics, University of Wisconsin, 325 Van Vleck Hall, \\ 480 Lincoln Drive, Madison, WI 53706, USA; \\ email: ellenber@math.wisc.edu \\ 2 Department of Mathematics, University of Pennsylvania, David Rittenhouse Lab., \\ 209 South 33rd Street, Philadelphia, PA 19104-6395, USA; \\ email:mhabli@math.upenn.edu
}

Received 22 August 2014; accepted 22 May 2016

\begin{abstract}
In this note we generalize a recent theorem of Guth and Katz on incidences between points and lines in 3-space from characteristic 0 to characteristic $p$, and we explain how some of the special features of algebraic geometry in characteristic $p$ manifest themselves in problems of incidence geometry.

2010 Mathematics Subject Classification: 52C10 (primary); 14A25 (secondary)

The goal of this note is twofold: first, to generalize a recent theorem of Guth and Katz on incidences between points and lines in 3-space from characteristic 0 to characteristic $p$, and second, to explain how some of the special features of algebraic geometry in characteristic $p$ manifest themselves in problems of incidence geometry.

Let $X$ be a reduced and irreducible hypersurface in $\mathbb{A}^{n}$, that is the variety cut out by the vanishing of some irreducible polynomial $F\left(x_{1}, \ldots, x_{n}\right)$. We say that $F$ is planar if, for every smooth point of $x$, the tangent plane to $X$ at $x$ meets $X$ with a degree of tangency greater than two. For instance, to say a plane curve is planar is to say that every smooth point on the curve is an inflection point. Of course, for a plane curve over the real numbers, this implies that the curve is a line. But this is not the case in characteristic $p$. For instance, the curve with equation $x^{3} y+y^{3} z+z^{3} x$ over $\mathbb{F}_{3}$ is planar; this is the curve famously called 'the funny curve' by Hartshorne.

(c) The Author(s) 2016. This is an Open Access article, distributed under the terms of the Creative Commons Attribution licence (http://creativecommons.org/licenses/by/4.0/), which permits unrestricted re-use, distribution, and reproduction in any medium, provided the original work is properly cited.
\end{abstract}


Since we are working over a field of characteristic $p$, the notions of 'tangency' and 'differential' relevant here are the algebraic versions. In particular, to say $X$ passes through the origin is to say $F$ vanishes at the origin, and thus has a Taylor series there that starts:

$$
F\left(x_{1}, \ldots, x_{n}\right)=0+F_{1}\left(x_{1}, \ldots, x_{n}\right)+F_{2}\left(x_{1}, \ldots, x_{n}\right)+\text { h.o.t. }
$$

where $F_{i}$ is a homogeneous polynomial of degree $i$. To say that $X$ is planar at the origin is precisely to say that $F_{2}$ is divisible by $F_{1}$. (The 'planar' points here are precisely those points which are called 'flat points' by Guth and Katz; we have avoided the word 'flat' here in order to avoid conflict with its other uses in arithmetic geometry.)

THEOREM 1. Let $k$ be a field and let $L$ be a set of $N^{2}$ lines in $k^{3}$, such that no $2 N d$ lines lie in any planar surface of degree $d$. Let $S$ be a set of points such that each line in L contains at least $N$ points of $S$. Then $|S|>c N^{3}$ for some absolute constant $c$.

REMARK 1. The notion of 'planarity' is very closely related to that of nonreflexivity, the failure of the map from $X$ to its dual variety to be generically smooth; indeed, for curves in odd characteristic the two notions are the same, as [3, Theorem 5.90] shows. We have chosen to use the less standard criterion of 'planarity' on the grounds that it is simpler to describe and fits more naturally into the proof of the theorem.

In characteristic 0 , every planar surface is a plane. In that case, Theorem 1 is an assertion about a family of $N^{2}$ lines, no $2 N$ of which are contained in any plane. This latter condition can be thought of as a form of the Wolff axiom. So, when $k$ has characteristic 0 , the conclusion of Theorem 1 is a theorem of Guth and Katz [2, Theorem 2], which settles a conjecture of Bourgain. (The theorem in [2] is stated for $k=\mathbb{R}$, but the proof works word for word over any field of characteristic 0.)

In characteristic $p$, however, Theorem 1 does not hold without the restriction concerning lines lying in a planar surface. For example, take $k=\mathbb{F}_{p^{2}}$ and let $X \subset \mathbb{A}^{3}$ be the 'Heisenberg surface' cut out by the equation

$$
x-x^{p}+y z^{p}-z y^{p}=0 .
$$

Then the lines of the form $\left\{(a, b, 0)+t(\bar{b}, v, 1) \mid t \in \mathbb{F}_{p^{2}}\right\}$, where $a$ and $v$ both lie in $\mathbb{F}_{p}$ and $\bar{b}$ denotes the Galois conjugate $b^{p}$ of $b$, lie in $X$. There are $p^{4}$ such lines; let $L$ be this set of lines. Now take $S$ to be the set $X\left(\mathbb{F}_{p^{2}}\right)$ and take $N=p^{2}$. 
The intersection of any plane with $X$ is a curve of degree at most $p+1$, which can contain at most $p+1$ (that is, approximately $N^{1 / 2}$ ) lines. But $S$ clearly contains all $N$ of the $\mathbb{F}_{p^{2}}$-rational points on each of the lines in $L$. Finally, one can check that $|S| \sim N^{5 / 2}$; so this set does not conform to the conclusion of Theorem 1 . However, $X$ is a planar surface of degree $N^{1 / 2}+1$, in which all $N^{2}$ of the lines are contained; so this counterexample is excluded by the hypothesis of Theorem 1 .

We note that the Heisenberg surface is precisely the one that appears in the paper of Mockenhaupt and Tao [5, Section 8] as an example of a set $P$ of points in 3-dimensional space over a finite field $\mathbb{F}$ which contains $|\mathbb{F}|^{2}$ lines, no $|\mathbb{F}|$ of which are contained in a plane, but which has cardinality much less than $|\mathbb{F}|^{3}$. The Mockenhaupt-Tao paper concerned Kakeya sets in $\mathbb{F}^{3}$ : subsets containing a line in each of the $\sim|\mathbb{F}|^{2}$ possible directions. It was conjectured by Wolff [6] that Kakeya sets have cardinality of order $|\mathbb{F}|^{3}$ in contrast to Kakeya sets in $\mathbb{R}^{3}$ which can have measure 0 . Kakeya sets in $\mathbb{F}^{3}$ are now known, by Dvir's theorem [1], to have cardinality of order $|\mathbb{F}|^{3}$. The Heisenberg surface shows that, by contrast, there are much smaller subsets of $\mathbb{F}^{3}$ which satisfy the Wolff axiom and which contain $|\mathbb{F}|^{2}$ lines, once we relax the condition that these lines all point in different directions.

In the important case where $k=\mathbb{F}$ is a finite field and $N=|\mathbb{F}|$, Theorem 1 can be seen as a strengthening of Dvir's theorem in the 3-dimensional case. Suppose $L$ is a set of $N^{2}$ lines in $\mathbb{F}^{3}$ which satisfies the Kakeya condition; the lines all point in distinct directions. We may think of $\mathbb{F}^{3}$ as being affine space embedded in projective space $\mathbb{P}^{3}(\mathbb{F})$. Take $H$ to be the plane at infinity; then the Kakeya condition can be rephrased as saying that the lines in $L$ intersect the plane $H$ at infinity in $N^{2}$ distinct points. If $X$ is a hypersurface of degree $d$, then any line in $L$ which is contained in $X$ must intersect $H$ somewhere on the curve $X_{0}=X \cap H$. Since $X_{0}$ is a degree- $d$ plane curve, it has at most $d(\mathbb{F} \mid+1)$ points; thus, at most $d(|\mathbb{F}|+1)$ of the lines can be contained in the hypersurface $X$. In particular, the Kakeya condition implies the conditions of Theorem 1. But the weaker conditions of Theorem 1 already suffice to guarantee that the union of the $|\mathbb{F}|^{2}$ lines contains a positive proportion of the points of $\mathbb{F}^{3}$. Our point of view is that the conditions of Theorem 1 should be thought of as the appropriate modification of the Wolff axiom to use in a characteristic $p$ context.

When $\mathbb{F}$ is a prime field $\mathbb{F}_{p}$, the situation is even more agreeable. We note that planar surfaces which are not planes have to have degree at least as great as the characteristic:

LEMMA 1. Let $k$ be a field and let $X \in \mathbb{A}^{n} / k$ be a (reduced, irreducible) planar hypersurface of degree $d>1$. Then $d \geqslant p$.

Proof. Without loss of generality we assume $k$ is algebraically closed. It is immediate that any hyperplane section of a planar variety is a planar subvariety. 
Choose a plane in $\mathbb{A}^{n}$ whose intersection with $X$ is an irreducible curve; then $C$ is a plane curve of degree $d>1$ which is planar, which is well known to have degree at least $p$ (see for example [3, Theorem 5.90]).

We remark that the real arithmetic content of Lemma 1 is that, when $X$ is planar, the map from $X$ to its dual is not generically smooth, which means that it must be inseparable, which means that its degree must be a multiple of $p$.

From Lemma 1 and Theorem 1 one immediately obtains the following corollary:

COROLlary 1 . Let $L$ be a set of $p^{2}$ lines in $\mathbb{F}_{p}^{3}$, no more than $2 p$ of which lie in any plane. Then the union of all the lines in L has cardinality at least $c p^{3}$ for some absolute constant $c$.

Proof. Apply Theorem 1 with $N=p$, noting that the second part of the hypothesis is vacuous, since $2 N d \geqslant 2 p^{2}>|L|$ for any planar surface which is not a plane.

In other words, the conclusion of Guth and Katz regarding incidences of lines and points over $\mathbb{R}$ remains true as an assertion about lines and points over $\mathbb{F}_{p}$, while it is false, as witnessed by the Heisenberg surface, when $k=\mathbb{F}_{p^{2}}$.

One might ask over which finite fields the analogue of Corollary 1 holds; in fact, it is true only for finite fields of prime order, as we now demonstrate.

Specifically, we will show that the hypersurface $X$ of $\mathbb{F}_{p^{n}}^{3}$ cut out by the polynomial

$x+x^{p}+\cdots+x^{p^{n-1}}+y z^{p}+y^{p} z^{p^{2}}+\cdots+y^{p^{n-1}} z-y z^{p^{n-1}}-y^{p} z-\cdots-y^{p^{n-1}} z^{p^{n-2}}$

contains $p^{2 n}$ lines, but $\left|X\left(\mathbb{F}_{p^{n}}\right)\right|=p^{3 n-1}$. We denote the polynomial by $f(x, y, z)$.

First of all, we note that the expression $x+\cdots+x^{p^{n-1}}$, as $x$ ranges over $\mathbb{F}_{p^{n}}$, takes each value in $\mathbb{F}_{p}$ exactly $p^{n-1}$ times. It follows that $f$, considered as a map from $\mathbb{F}_{p^{n}}^{3}$ to $\mathbb{F}_{p}$, takes each value $p^{3 n-1}$ times. In particular, $\left|X\left(\mathbb{F}_{p^{n}}\right)\right|=p^{3 n-1}$. Next, we show that the surface contains at least $p^{2 n}$ lines. We are only interested in lines which intersect the $x y$-plane transversely, that is, which are of the form $L_{(a, b, u, v)}=\left\{(a, b, 0)+t(u, v, 1) \mid t \in \mathbb{F}_{p^{n}}\right\}$. Note that the values of $a, b, u, v$ uniquely determine the line.

If $L_{(a, b, u, v)} \subset X$, then the triples $x=a+t u, y=b+t v, z=t$ are solutions for $f(x, y, z)$ for any value of $t$. Therefore, the coefficients of the $t^{j}$ 's vanish. It is straightforward to check that the coefficients $c_{l}$ of $t^{l}$ can be characterized as follows:

- if $l=p^{j}+p^{j-1}$, then $c_{l}=c_{p^{j}+p^{j-1}}=v^{p^{j-1}}-v^{p^{j}}$;

- if $l=p^{j}$, then $c_{l}=c_{p^{j}}=b^{p^{j-1}}-b^{p^{j+1}}+u^{p^{j}}$; 
- if $l=0$, then $c_{l}=c_{0}=a+a^{p}+\cdots+a^{p^{n-1}}$; and

- $c_{l}=0$, otherwise.

Note that $c_{p^{i}}^{p}=c_{p^{i+1}}$, therefore $c_{p^{i}}$ vanishes if and only if $c_{p^{j}}$ vanishes. Similarly, $c_{p^{i}+p^{i-1}}=0$ if and only if $c_{p^{i+1}+p^{i}}=0$. As a consequence $L_{(a, b, u, v)} \subset X$ if and only if

- $v-v^{p}=0$;

- $b-b^{p^{2}}+u^{p}=0$;

- $a+a^{p}+\cdots a^{p^{n-1}}=0$.

So a line is given by one of the $p$ choices for $v$, one of the $p^{n}$ choices for $b$ (which determines $u$ ) and one of the $p^{n-1}$ choices for $a$. So the number of lines of the form $L_{(a, b, u, v)}$ contained in $X$ is $p \cdot p^{n} \cdot p^{n-1}=p^{2 n}$. (It is easy to show that no more than $2 p$ such lines lie in a plane.)

QUESTION 1. Arguing as above, one can show that, if $q$ is a prime power $p^{m}$ with $m>1$, there is a set of $q^{2}$ lines in $\mathbb{F}_{q}^{3}$, no $q$ contained in a plane, whose union has cardinality $\sim q^{3-1 / d}$, where $d$ is the smallest nontrivial divisor of $m$. Is this sharp? (The argument of Mockenhaupt and Tao shows that the union can be no smaller than $q^{5 / 2}$, so the bound is sharp when $m$ is even.) This question might be approachable by a more refined description of planar surfaces of low degree.

REMARK 2. A recent preprint by János Kollár [4], appearing after the present paper was completed, proves a Szemerédi-Trotter theorem in three dimensions over arbitrary fields, and from there derives a separate (but similar in spirit) proof of Corollary 1.

We now prove Theorem 1 .

Proof. The following lemma is unchanged from Guth-Katz (see the proof of Theorem 1.2 of [2]), which we add for the sake of completeness.

LEMMA 2. Let $L$ be a set of $N^{2}$ lines in $k^{3}$ and let $S$ be a set of points such that each line in $L$ contains at least $N$ points of $S$. Suppose $|S|=N^{3} / K$, where $K$ is a sufficiently large constant. Then, there exists an irreducible hypersurface $X$ of degree $d$, a subset $S^{\prime}$ of the intersection of $S$ and $X(k)$, and nested subsets $L^{\prime \prime} \subset L^{\prime} \subset L$ such that

- each line in $L^{\prime}$ contains at least $100 d$ points of $X(k)$, (and hence $L^{\prime}$ is contained in $X)$;

- each point of $S^{\prime}$ is on at least 3 lines of $L^{\prime}$; 
- each line in $L^{\prime \prime}$ contains at least $10 d$ points of $S^{\prime}$; and

- $\left|L^{\prime \prime}\right| \geqslant 2 N d \geqslant 4 d^{2}$.

Proof. We assume, again without loss of generality, that $k$ is algebraically closed.

If $I$ is the set of incidences, that is the set of pairs $(p, \ell)$ with $p$ a point in $S$ and $\ell$ a line in $L$ containing $p$, then $I$ contains at least $N$ points projecting to any given line. We distinguish a subset $I^{\prime}$ of $I$ containing exactly $N$ incidences for each line, and from now on use the word 'incidence' to refer only to these distinguished incidences. In particular, if $T$ is a subset of $S$ and $M$ a subset of $L$, we denote by $I(T, M)$ the number of incidences $(p, \ell)$ in $I^{\prime}$ with $p \in T$ and $\ell \in M$. So $I(S, L)=|L| \cdot N=N^{3}$.

We define $v(x)$ to be the number of lines incident to $x$. We denote by $S_{v}$ the set of points $x$ of $S$ such that $v(x) \geqslant K / 1000$. Each line is incident to exactly $N$ points, therefore

$$
I\left(S \backslash S_{v}, L\right) \leqslant|S| \cdot \frac{K}{1000}=\frac{N^{3}}{K} \cdot \frac{K}{1000}=\frac{N^{3}}{1000}
$$

implying that $I\left(S_{v}, L\right) \geqslant 999 N^{3} / 1000$.

We define similarly the sets $S_{j}$ to be the sets of points $x \in S$ such that $2^{j-1} K / 1000 \leqslant v(x) \leqslant 2^{j} K / 1000$. Since, $\sum_{j=1}^{\infty} I\left(S_{j}, L\right) \geqslant I\left(S_{v}, L\right) \geqslant$ $999 N^{3} / 1000$ and $\sum_{j=1}^{\infty}\left(1 / j^{2}\right)<2$, by the pigeonhole principle, there exists a $j \geqslant 1$ such that $I\left(S_{j}, L\right) \geqslant 999 N^{3} / 2000 j^{2}$. Since for each element $x$ of $S_{j}$, $v(x) \leqslant 2^{j} K / 1000$, we obtain

$$
\left|S_{j}\right| \cdot \frac{2^{j} K}{1000} \geqslant I\left(S_{j}, L\right) \geqslant \frac{999 N^{3}}{2000 j^{2}},
$$

implying that

$$
\left|S_{j}\right| \geqslant \frac{999 N^{3}}{2 K 2^{j} j^{2}} .
$$

Similarly, since $2^{j-1} K / 1000 \leqslant v(x)$ for each element of $S_{j}$, thus $N^{3}=$ $I(S, L) \geqslant I\left(S_{j}, L\right) \geqslant\left|S_{j}\right| \cdot 2^{j-1} K / 1000$, implying

$$
\frac{2000 N^{3}}{K 2^{j}} \geqslant\left|S_{j}\right| \geqslant \frac{999 N^{3}}{2 K 2^{j} j^{2}} .
$$

For any set $T \subset k^{3}$ of size at most $\left(\begin{array}{c}d+3 \\ 3\end{array}\right)$, there exists a polynomial of degree at most $d$ vanishing on the points of $T$. Since $\left|S_{j}\right| \leqslant 2000 N^{3} / K 2^{j}$, there exists a polynomial $P$ of degree at most $25 N / K^{1 / 3} 2^{j / 3}$ vanishing on $S_{j}$. We can assume 
that $P$ is square-free and separable. It may not be irreducible; if it is not, we may factor it into irreducible factors, $P=P_{1} P_{2} \ldots P_{m}$. We denote the degrees of the $P_{l}$ by $d_{l}$. Let $S_{j, l}$ be the set of points of $S_{j}$ where $P_{l}$ vanishes. We have

$$
\sum_{l=1}^{m} d_{l} \leqslant \frac{25 N}{K^{1 / 3} 2^{j / 3}} \quad \text { and } \quad \sum_{l=1}^{m}\left|S_{j, l}\right| \geqslant \frac{999 N^{3}}{2 K 2^{j} j^{2}}
$$

Again, by the pigeonhole principle, we can find an $l$ such that

$$
\left|S_{j, l}\right| \geqslant \frac{999 N^{2} d_{l}}{50 K^{2 / 3} 2^{2 j / 3} j^{2}}
$$

We denote by $X$ the hypersurface cut out by $P_{l}$, and by $d$ the corresponding degree $d_{l}$. Note that $S_{j, l} \subset X(k)$.

We denote by $L^{\prime}$ the set of lines in $L$ incident to more than $100 d$ points of $X(k)$. Clearly, $L^{\prime} \subset L \cap X(k)$ and

$$
I\left(S_{j, l}, L \backslash L^{\prime}\right) \leqslant\left|L^{\prime}\right| 100 d \leqslant|L| 100 d=100 N^{2} d .
$$

A similar calculation shows that if $S^{\prime}$ denotes the set of points of $S_{j, l}$ incident to at least 3 lines of $L^{\prime}$, then we have $I\left(S_{j, l} \backslash S^{\prime}, L^{\prime}\right) \leqslant 2\left|S_{j, l}\right|$. Finally, if $L^{\prime \prime}$ denotes the set of lines in $L^{\prime}$ incident to more than $10 d$ points of $S^{\prime}$, then $I\left(S^{\prime}, L^{\prime} \backslash L^{\prime \prime}\right) \leqslant$ $|L| 10 d=10 N^{2} d$. Combining the above inequalities we have

$$
\begin{aligned}
I\left(S^{\prime}, L^{\prime \prime}\right) & \geqslant I\left(S_{j, l}, L\right)-I\left(S_{j, l}, L \backslash L^{\prime}\right)-I\left(S_{j, l} \backslash S^{\prime}, L^{\prime}\right)-I\left(S^{\prime}, L^{\prime} \backslash L^{\prime \prime}\right) \\
& \geqslant\left|S_{j, l}\right| \frac{2^{j-1} K}{1000}-100 N^{2} d-2\left|S_{j, l}\right|-10 N^{2} d .
\end{aligned}
$$

By definition $\left|S_{j, l}\right| \geqslant 999 N^{2} d / 50 K^{2 / 3} 2^{2 j / 3} j^{2}$, which implies that

$$
\left|S_{j, l}\right| \frac{2^{j-1} K}{1000} \geqslant \frac{999 N^{2} d \cdot 2^{j / 3} K^{1 / 3}}{100 j^{2} \cdot 1000} .
$$

Notice that $2^{j / 3} / j^{2}$ is bounded from below, hence we can choose a sufficiently large $K$ independent of $j$ so that $I\left(S^{\prime}, L^{\prime \prime}\right) \geqslant 2 N^{2} d$. Since every line in $L^{\prime \prime}$ is incident to at most $N$ points of $S^{\prime}$, we obtain $\left|L^{\prime \prime}\right| \geqslant 2 N d$. 
By taking a possibly larger $K$, we can ensure that $d \leqslant N / 4$, and thus $\left|L^{\prime \prime}\right| \geqslant$ $2 N d \geqslant 4 d^{2}$.

LEMMA 3. Let $X$ be a reduced irreducible nonplanar surface of degree $d>1$ in $\mathbb{A}^{3}$. Let $L_{1}$ be the set of lines contained in $X$ which contain at least d singular points of $X$, and let $L_{2}$ be the set of lines contained in $X$ which contain at least $3 d-3$ planar points of $X$. Then

$$
\left|L_{1}\right|+\left|L_{2}\right|<4 d^{2}
$$

Proof. Let $F$ be an irreducible square-free polynomial such that $V(F)=X$.

The singular locus of $X$ is cut out by the vanishing of $F$ and its first partial derivatives, which are of degree $d-1$. So $F$ and all the partial derivatives vanish identically on every line in $L_{1}$. Since $X$ is reduced, the singular locus is a curve $C_{1}$ in $X$, and since $C_{1}$ is contained in the intersection of $X$ with one of its partial derivatives, we have $\operatorname{deg} C_{1} \leqslant d(d-1)$. In particular, no more than $d(d-1)$ lines can be contained in $C_{1}$, so $\left|L_{1}\right| \leqslant d(d-1)$.

Let $C_{2}$ be the locus of planar points of $X$. By hypothesis, $C_{2}$ is a proper subvariety of $X$. Let $p$ be a nonplanar point of $X$; then the generic hyperplane section of $X$ containing $p$ is a curve which is not planar. Choose a hyperplane $H$ such that $H \cap X$ is a nonplanar curve $Z$ in $H$. By change of coordinates, we may assume that $p$ is the origin and $H$ is the plane $z=0$. So $Z$ is simply the plane curve cut out by the vanishing of $F(x, y, 0)$. At any planar point $(a, b, 0)$ of $Z$, the Taylor series of $F(x, y)$ starts

$$
F(x, y)=F_{1}(x, y)+F_{2}(x, y)+\text { h.o.t. }
$$

where

$$
F_{1}(x, y)=F_{x}(x-a)+F_{y}(y-b)
$$

and

$$
F_{2}(x, y)=\frac{F_{x x}(x-a)^{2}}{2}+F_{x y}(x-a)(y-b)+\frac{F_{y y}(y-b)^{2}}{2}
$$

(where $F_{x}$ (respectively $F_{x y}$ ) denotes the partial derivative (respectively second partial derivative) of $F$ with respect to $x$ (respectively with respect to $x, y$ ).) In characteristic 2 , we define $F_{x x} / 2$ to be the divided power operation; in other words, $x_{x x}^{n} / 2$ is defined to be $(n(n+1) / 2) x^{n-2}$. Since $(a, b, 0)$ is a planar point of $Z, F_{2}$ is divisible by $F_{1}$. For a smooth point $(a, b, 0), F_{2}$ is divisible by $F_{1}$ if and only if the matrix

$$
\left(\begin{array}{ccc}
F_{x} & 0 & F_{x x} \\
F_{y} & F_{x} & F_{x y} \\
0 & F_{y} & F_{y y}
\end{array}\right)
$$


is singular. In other words the polynomial

$$
G:=F_{x}^{2} \frac{F_{y y}}{2}+F_{y}^{2} \frac{F_{x x}}{2}-F_{x} F_{y} F_{x y}
$$

vanishes only at those smooth points which are planar.

The locus $C_{2}$ is contained in the locus where both $F$ and $G$ vanish, and the nonplanarity of $Z$ implies that $G$ is not a multiple of $F$; thus $F \cap G$ is a curve of degree $(\operatorname{deg} F)(\operatorname{deg} G)=d(3 d-4)$, which contains $C_{2}$. The restriction of $G$ to any line in $L_{2}$ is a polynomial of degree $3 d-4$ which vanishes at at least $3 d-3$ distinct points, and is thus 0: so all the lines in $L_{2}$ are contained in the curve $F \cap G$, which implies that $\left|L_{2}\right| \leqslant d(3 d-4)$.

REMARK 3. A direct consequence of Lemma 3 is that the number of lines which contain at least $4 d-3$ either singular or planar points of a reduced irreducible nonplanar surface $X$ of degree $d$ is less than $4 d^{2}$.

Set $|S|=N^{3} / K$ and assume $K$ is large enough to apply Lemma 2. Using Lemma 2, we obtain an irreducible hypersurface $X$ of degree $d$ and sets $L^{\prime}, S^{\prime}$ and $L^{\prime \prime}$. Each line in $L^{\prime}$ contains at least $100 d$ points of $X$, and is thus contained in $X$ as a variety. Each point $p$ in $S^{\prime}$ is contained in at least three such lines; either these three lines lie in a plane, implying that $p$ is a planar point of $X$, or the direction vectors of the lines span the whole space, implying that $p$ is a singular point of $X$. Therefore, each point in $S^{\prime}$ is either a singular or a planar point of $X$. Furthermore, $\left|L^{\prime \prime}\right| \geqslant 2 N d \geqslant 4 d^{2}$ and each line in $L^{\prime \prime}$ contains at least $10 d$ points of $S^{\prime}$. By Lemma 3, $X$ is a planar surface. But $X$ contains at least $2 N d$ lines of $L$, which violates the hypothesis of the theorem.

\section{References}

[1] Z. Dvir, 'On the size of Kakeya sets in finite fields', J. Amer. Math. Soc. 22(4) (2009), 1093-1097.

[2] L. Guth and N. Katz, 'Algebraic methods in discrete analogs of the Kakeya problem', Adv. Math. 225(5) (2010), 2828-2839.

[3] J. W. P. Hirschfeld, G. Korchmáros and F. Torres, Algebraic Curves over a Finite Field (Princeton University Press, Princeton, NJ, 2013).

[4] J. Kollár, 'Szemerédi-Trotter-type theorems in dimension 3', Preprint, 2014, arXiv:1405.2243v2.

[5] G. Mockenhaupt and T. Tao, 'Restriction and Kakeya phenomena for finite fields', Duke Math. J. 121(1) (2004), 35-74.

[6] T. Wolff, 'Recent work connected with the Kakeya problem', in Prospects in Mathematics (Princeton, NJ, 1996) (American Mathematical Society, Providence, RI, 1999), 129-162. 